\title{
Effectiveness of Short Wave Diathermy and Acupuncture with Supervised Active Exercise for the Treatment of Frozen Shoulder: A Case Report
}

\author{
Manpreet Kaur Bhamra' ${ }^{1}$, Jasraj Kaur Bhamra ${ }^{1}$, Waqar M. Naqvi ${ }^{2}$ and Gaurav Mishra ${ }^{3}$ \\ ${ }^{1}$ Community Health Physiotherapy, Ravi Nair Physiotherapy College, Datta \\ Meghe Institute of Medical Sciences, Wardha, Maharashtra, India \\ ${ }^{2}$ Head of Department, Community Health Physiotherapy, Ravi Nair Physiotherapy \\ College; Research Consultant, Jawaharlal Nehru Medical College, Datta Meghe \\ Institute of Medical Sciences, Wardha, Maharashtra, India \\ ${ }^{3}$ Department of Radiodiagnosis, Jawaharlal Nehru Medical College, Datta Meghe \\ Institute of Medical Sciences, Sawangi (M), Wardha
}

\section{ABSTRACT}

A disabling condition causing glenohumeral pain and lack of mobility is the frozen shoulder. Frozen shoulder-related pain and reduced mobility impair the functioning of the patient, making therapeutic intervention possible. Frozen shoulder affects around 2-5 percent of the population, most commonly around fifty years of age. Females are around 58 percent more prone to get affected by this condition. Case Presentation: A 54-year-old woman was diagnosed with right frozen shoulder. She had history of painful joint with limited range of motionof glenohumeral joint for four-months. The range of motion of right shoulder was limited. Apart from medicinal treatment,physiotherapy involving short-wave diathermy, acupuncture and controlled active frozen shoulder management exercises was given. Shortwave diathermy and supervised active exercises increase the frozen shoulder resolution rate.Acupuncture has been shown to be a safe procedure with a positive impact in terms of pain relief and shoulder function recovery.

\section{KEY WORDS: FROZEN SHOULDER, PAINFUL SHOULDER JOINT, SHORTWAVE DIATHERMY, ACUPUNCTURE.}

\section{INTRODUCTION}

The shoulder is a special anatomical structure with a complex range of motion that helps us to work in our environment effectively. A disabling condition causing glenohumeral pain and lack of mobility is the frozen shoulder. Frozen shoulder-related pain and reduced mobility impair the functioning of the patient, making therapeutic intervention possible (Anthonia et al., 2020)

Biosc Biotech Res Comm P-ISSN: 0974-6455 E-ISSN: 2321-4007

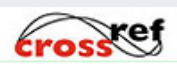

Identifiers and Pagination

Year: 2021 Vol: 14 No (6) Special Issue

Pages: $77-80$

This is an open access article under Creative

Commons License Attribn 4.0 Intl (CC-BY). DOI: $h t t p: / / d x . d o i . o r g / 10.21786 / b b r c / 14.6 .18$
Frozen shoulder is also known as "adhesive capsulitis" or"periarthritis". This condition affects mainly around the age of fifty and occurs in 2-5 percent of the population. Pain usually aggravates at night and can affect sleep quality. Females have 58 percent higher risk for this condition. Frozen shoulder is characterised by three phases namely freezing, frozen, and thawing phase (BenArie et al., 2020) Patients with frozen shoulder generally report with gradual onset of pain and loss of active and passive shoulder motions in elevation and rotation. The presence of multiregional synovitis with inflammation is usually a marked feature of Frozen shoulder ( Anthonia et al., 2020).

Arthroscopic findings reflect inflammation in the anterosuperior joint capsule, axillary fold of the joint capsule. Also the coracohumeral ligament and rotator cuff interval gets affected frequently. Inflammation then

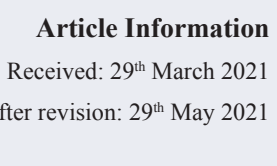

Article Information
Received: $29^{\text {th }}$ March 2021 ccepted after revision: 29th May 2021 
progresses to fibrosis and adhesion (Ben-Arie et al., 2020) Recent evidence indicates that elevated serum cytokine levels in patients with frozen shoulder induce sustained severe and prolonged inflammatory response affects the capsuloligamentous complex along with synovial lining ( Anthonia et al., 2020) The history and physical examination of the patient is generally adequate to diagnose frozen shoulder ( Ben-Arie et al., 2020).

On the other hand, shortwave diathermy given at a comfortable heating strength by $27.12 \mathrm{MHz}$ wave through anterior and posterior electrodes as an alternative therapy to stretching exercises led to substantial improvement in pain and range of motion. Physiotherapy's primary purpose is to alleviate discomfort, increase movement range, strengthen weak muscles and regain optimum function (Anthonia et al., 2020) Acupuncture can relieve pain and enhance the function of the ROM and the shoulder (Ben-Arie et al., 2020).

Patient And Observation: A 54 year old woman,with BMI 27.6 reported with an insidious onset of right shoulder pain. There was steady rise in pain and a gradual reduction in the active and passive range of motion on right shoulder. Grooming activities, conducting overhead tasks, dressing and particularly fastening objects behind the back became difficult. There was more than 25\% loss of range of motion, especially abduction and external rotation (Anthonia et al., 2020) Palpation around the right shoulder caused higher anterior pain during physical testing, and pain increased as the right shoulder moved.

Limited movement was observed in all directions, particularly in abduction and external rotation. Mild muscle tightness was noted around the right shoulder. No apparent muscle atrophy or differential warmth was found (Anthonia et al., 2020) There is decreased muscle strength of right side. She visited to physiotherapy department. Around 8 weeks therapy with shortwave diathermy, along with acupuncture and controlled active exercises helped to reduce pain and increase range of motion.Electroacupuncture (EA) showed beneficial effect in pain relief for short term pain (Ben-Arie et al., 2020).

Table 1. Manual muscle testing (strength) assessment on 1 day of treatment.

\begin{tabular}{|l|c|c|}
\hline \multirow{2}{*}{ Joints } & & pre-assessment \\
\hline \multirow{2}{*}{ Shoulder } & Flexor & 2 \\
\cline { 2 - 3 } & Extensor & 3 \\
\cline { 2 - 3 } & abductor & 2 \\
\hline \multirow{2}{*}{ Elbow flexor } & Extensor & 4 \\
& & 4 \\
\hline
\end{tabular}

Clinical Finding: Patient is diagnosed with decreased range of motion, weakness and decreased muscle strength of right side shoulder[Table 1 and Table 2].
Table 2. Range of motion of joints on 1 day of treatment.

\begin{tabular}{|l|c|c|}
\hline Joint & Active & Passive \\
\hline Shoulder flexion & $0-117$ & $0-125$ \\
\hline Extension & $0-35$ & $0-35$ \\
\hline Abduction & $0-98$ & $0-100$ \\
\hline External rotation & $0-18$ & $0-23$ \\
\hline Internal rotation & $0-15$ & $0-20$ \\
\hline Elbow flexion & $0-137$ & $0-140$ \\
\hline extension & 0 & 0 \\
\hline
\end{tabular}

Diagnostic Assessment: Shortwave diathermy, acupuncture and controlled active exercises were done after orthopaedic examination. This showed restricted range of motion and muscle weakness of right shoulder.

Theraputic Interventation:

1. Modality: Short wave diathermy

2. Acupuncture

3. Controlled passive movement

4. Controlled active movement

Follow Up And Outcome: Frozen management requires a mixture of pharmacological, rehabilitative, and/or surgical therapy. These involve the use of NSAIDS, corticosteroids, anaesthesia manipulation, physiotherapy, osteopathy, occupational therapy and capsular arthroscopic release for frozen shoulder management (Anthonia et al., 2020).

Modalities of physiotherapy that can be used to alleviate frozen shoulder include:

1. Transcutaneous electrical stimulation[TENS]

2. Ultrasound

3. Short wave diathermy

4. Low level laser therapy

5. Hot and cold compression packs

6. Active exercise

7. Passive exercise

8. Acupuncture

9. Interferential current

10. Pulse electromagnetic field therapy

Table 3. MMT of upper-extremity pre and post assessment

\begin{tabular}{|l|c|c|c|}
\hline \multirow{2}{*}{ Assessment } & \multicolumn{1}{|c|}{$\begin{array}{c}\text { Pre- } \\
\text { assessment }\end{array}$} & $\begin{array}{c}\text { Post- } \\
\text { assessment }\end{array}$ \\
\hline \multirow{3}{*}{ Shoulder } & Flexor & 2 & 4 \\
\cline { 2 - 4 } & Extensor & 3 & 5 \\
\cline { 2 - 4 } & abductor & 2 & 4 \\
\hline \multirow{2}{*}{ Elbow } & flexor & 4 & 5 \\
\cline { 2 - 4 } & Extensor & 4 & 5 \\
\hline
\end{tabular}


Short wave diathermy as one of the electrotherapy approaches used in frozen shoulder management, shortwave diathermy induces it physiological and therapeutic effects by rapidly alternating electrical and magnetic currents at short wave frequencies [27.12hHz]. (Anthonia et al., 2020). It can be pulsed or constant. Shortwave diathermy utilise high frequency oscillating electromagnetic field to heat surfaces of the body. It heats up to a depth of 2 to $3 \mathrm{~cm}$ of tissue. It was applied at a comfortable heating power by two electrodes placed anteriorly and posteriorly on the patient's shoulder (40watts). Its purpose is to alleviate pain and enhance the extensibility of tissue (Anthonia et al., 2020)

\begin{tabular}{|c|c|c|c|c|}
\hline \multirow{2}{*}{\multicolumn{3}{|c|}{$\begin{array}{l}\text { Table 4. Range of motion of upper- } \\
\text { assessment. } \\
\begin{array}{l|l}\text { Assessment } & \text { Pre- assessment } \\
\text { Joint } & \text { Active Passive }\end{array}\end{array}$}} & \multicolumn{2}{|c|}{ Post-assessment } \\
\hline & & & Active & Passive \\
\hline Shoulder flexion & $0-117$ & $0-125$ & $0-160$ & $0-175$ \\
\hline extension & $0-35$ & $0-35$ & $0-50$ & $0-60$ \\
\hline Abduction & $0-98$ & $0-100$ & $0-145$ & $0-150$ \\
\hline External rotation & $0-18$ & $0-23$ & $0-65$ & $0-75$ \\
\hline Internal rotation & $0-15$ & $0-20$ & $0-50$ & $0-75$ \\
\hline Elbow flexion & $0-137$ & $0-140$ & $0-140$ & $0-140$ \\
\hline Extension & 0 & 0 & 0 & 0 \\
\hline
\end{tabular}

The supervised active exercises used in this research include:

1. Pendulum stretch

2. Towel stretch

3. Finger walk

4. Cross body reach

5. Armpit stretch

6. Outward rotation

7. Inward rotation

Every exercise should consist 10 repetition daily.

Immediate pain relief was observed by using press tack acupuncture needles versus press tack placebo (Ben-Arie et al., 2020) After 8 weeks, patient experienced improved range of motion and muscle strength.

\section{DISCUSSION}

Frozen shoulder is a self-limiting disorder that is found primarily in the diabetic population, especially among women. When the cause is identified or results from a surgical case, it may be primary (idiopathic) or secondary. There are three subcategories of secondary frozen shoulder that include systemic, extrinsic and intrinsic causes. This research used a physiotherapy technique involving short-wave diathermy, acupuncture and controlled active frozen shoulder management exercises (Anthonia et al., 2020). Studies on shoulder pain and joint morphology diagnostics and treatment were reviewed (Meshram et al., 2020; Saoji et al., 2020; Singh et al., 2019; Deshpande et al., 2018). Other related studies on physiotherapy rehabilitation were reported by Deshpande et al. (2014), Deshmukh et al. (2020), Ghordadekar et al (2020) and Shah et al. (2020). We suggest acupuncture trials to rely on reliable instruments such as SPADI, Arm, Shoulder and Hand Disabilities (DASH) for shoulder function measurements (Ben-Arie et al., 2020).

\section{CONCLUSION}

This research has shown that shortwave diathermy and supervised active exercises increase the frozen shoulder resolution rate (Anthonia et al., 2020). Acupuncture has been shown to be a safe procedure with a positive impact in terms of pain relief, shoulder function recovery, and short-term and mid-term flexion ROM (Ben-Arie et al., 2020).

Conflict of Interest: Nil.

Funding Support: None

\section{REFERENCES}

Anthonia, E.U., Ozoemene, E.V., Paulinus, I.C., Anthoninus, E.O., Eneje, E.L., Nkemdilim, O.C., Ekezie, U., Ikechukwu, E.C. and Chidiebere, O., Response Of Frozen Shoulder To Six Weeks Of Short Wave Diathermy And Supervised Active Exercises: A Case Report.

Asheghan, M., Aghda, A.K., Hashemi, E. and Hollisaz, M., 2016. Investigation of the effectiveness of acupuncture in the treatment of frozen shoulder. Materia sociomedica, 28(4), p.253.

Ben-Arie, E., Kao, P.Y., Lee, Y.C.,Ho, W.C., Chou, L.W., Liu, H.P. 2020. The Effectiveness of Acupuncture in the Treatment of Frozen Shoulder: A Systematic Review and Meta-Analysis. Evidence-Based Complementary and Alternative Medicine.pp.1-14.

Celik, D., 2010. Comparison of the outcomes of two different exercise programs on frozen shoulder. Acta orthopaedica et traumatologica turcica, 44(4), pp.285292.

Cheing, G.L., So, E.M. and Chao, C.Y., 2008. Effectiveness of electroacupuncture and interferential electrotherapy in the management of frozen shoulder. Journal of 
rehabilitation medicine, 40(3), pp.166-170.

Crubbs, N., 1993. Frozen shoulder syndrome: a review of literature. Journal of Orthopaedic \& Sports Physical Therapy, 18(3), pp.479-487.

Deshmukh, M.K., Fating, T.B. and Phansopkar, P.A., 2020. Comprehensive Physiotherapy Rehabilitation on a Complex Case of Combination of Subcoracoid, Subacromial, Subdeltoid, and Supraspinatus Tendinitis. Journal of Evolution of Medical and Dental Sciences, 9(41), pp.3075-3079.

Deshpande, S., Samal, N., Gawande, V. and Rathi, R., 2014. Bilateral Anterior Fracture Dislocation of Shoulder-A Rare case. Journal of Krishna Institute of Medical Sciences (JKIMSU), 3(1).

Deshpande, S.V., Phatak, S.V., Marfani, G.B., Gupta, N.A., Daga, S.S. and Samad, S.S., 2018. Sonographic evaluation of painful shoulder and its comparison with clinical diagnosis. Journal of Datta Meghe Institute of Medical Sciences University, 13(1), p.12.

Duenas, L., Balasch-Bernat, M., Aguilar-Rodriguez, M., Struyf, F., Meeus, M. and Lluch, E.A., 2019. 12-week tailored manual therapy and home stretching program based on level of irritability and range of motion impairments in patients with primary frozen shoulder contracture syndrome: a case series with 9-months follow-up. J Orthop Sports Phys Ther, 49(3), pp.192201.

Jawade, S. and Jawade, S., 2020. A Case report on impact of physiotherapy rehabilitation on partial claw hand secondary to borderline tuberculoid Hansen disease. European Journal of Molecular \& Clinical Medicine, 7(2), pp.1983-1986.

Latchoumi, T.P., Ezhilarasi, T.P. and Balamurugan, K., 2019. Bio-inspired weighed quantum particle swarm optimization and smooth support vector machine ensembles for identification of abnormalities in medical data. SN Applied Sciences, 1(10), pp.1-10.

Meshram, P., Pawaskar, A. and Kekatpure, A., 2020. 3D CT scan-based study of glenoid morphology in Indian population: Clinical relevance in design of reverse total shoulder arthroplasty. Journal of Clinical Orthopaedics and Trauma, 11, pp.S604-S609.

Saoji, K.K., Gawande, V. and Dulani, R., 2020. A Comparative study of Disability and Pain Assessment by Shoulder Pain and Disability Index (SPADI) Score in Patients of Adhesive Capsulitis Treated by Hydrodilatation with and without Corticosteroids. Int J Cur Res Rev| Vol, 12(14).

Shah, P.A., Nemade, S.V. and Naqvi, W.M., 2020. Advance Physiotherapeutic Rehabilitation Approach for Hand Functions in a Giant Cell Tumour Patient-A Case Report on Palliative Physiotherapy. Journal of Evolution of Medical and Dental Sciences, 9(21), pp.1675-1679.

Singh, R. and Singam, A., 2019. Comparison between Supraclavicular and Interscalene Brachial Plexus Block in Patients Undergoing Shoulder Surgery. Journal of Datta Meghe Institute of Medical Sciences University, 14(3), p.175. 